\title{
GLI INTERVENTI DI RESTAURO E DI ABBELLIMENTO DELLA CHIESA DI SANTA MARIA DEI GRECI DAL PERIODO NORMANNO AL BORBONICO
}

\author{
ROSANNA MAGRÌ \\ Università di Palermo
}

\begin{abstract}
La storia dei restauri e delle opere di "abbellimento" eseguiti nella chiesa di Santa Maria dei Greci in Agrigento attraversa un intervallo temporale che va dal periodo normanno ai nostri giorni, in un continuo lavoro di ricerca voluto da studiosi, che hanno creduto fortemente nella valorizzazione del monumento. La chiesa - sorta sui resti del tempio dorico risalente alla seconda metà del V secolo a. C.- è edificata con molta probabilità durante il periodo bizantino tra il 395 - Editto di Teodosio - e il 596, quindi prima della conquista musulmana. La struttura architettonica bizantina della chiesa ha subito, nel corso dei secoli, importanti trasformazioni che hanno determinato quella che oggi è la sua conformazione attuale. L'esame del periodo, che abbraccia quasi sette secoli - dal 1100 al 1750 - durante il quale sono state apportate all'edificio importanti trasformazioni architettoniche e completamenti stilistici, costituisce l'oggetto della presente argomentazione.
\end{abstract}

Parole chiave

Storia del restauro - Santa Maria dei Greci - Sicilia - Agrigento.

\section{RESTAURACION Y EMBELLECIMIENTO DE LA IGLESIA DE SANTA MARIA DEI GRECI DESDE EL PERIODO NORMANDO HASTA EL BORBON.}

\section{Resumen}

La historia de las obras de restauración y de las obras de "embellecimiento" llevadas a cabo en la iglesia de santa Maria dei Greci en Agrigento atraviesa un intervalo temporal que va desde el período normando hasta nuestros días, en una investigación continua realizada por estudiosos, quienes creyeron firmemente en la mejora del monumento. La iglesia, construida sobre los restos del templo dórico que data de la segunda mitad del siglo V a.C., (probablemente se construyó durante el período bizantino, entre 395, Edicto de Teodosio) y 596, antes de la conquista musulmana. La estructura arquitectónica bizantina de la iglesia ha sufrido, a lo largo de los siglos, importantes transformaciones que han determinado lo que hoy es su conformación actual. El examen del período que abarca casi siete siglos, desde 1100 hasta 1750 , durante el cual se han realizado importantes transformaciones arquitectónicas y terminaciones estilísticas en el edificio, es el objeto de este argumento

Palabras clave
Historia de las obras de restauración - Santa Maria dei Greci - Sicilia - Agrigento. 


\section{RESTORATION AND EMBELLIS HMENT OF THE CHURCH OF SANTA MARIA DEI GRECI FROM THE NORMAN PERIOD TO THE BOURBON.}

Abstract

The history of the restoration works and of the "embellishment" works carried out in the church of santa Maria dei Greci in Agrigento crosses a temporal interval that goes from the Norman period to our days, in a continuous research carried out by scholars, who strongly believed in the enhancement of the monument. The church - built on the remains of the doric temple dating back to the second half of the 5th century BC.C. - it is probably built during the Byzantine period between 395 - Edict of Theodosius - and 596, then before the Muslim conquest. The Byzantine architectural structure of the church has undergone, over the centuries, important transformations that have determined what today is its current conformation. The examination of the period, which spans almost seven centuries - from 1100 to 1750 - during which some important architectural transformations and stylistic completions have been made to the building, is the object of this argument.

$$
\text { Keywords }
$$

History of the restoration - Santa Maria dei Greci - Sicily - Agrigento.

\section{Premessa}

La più antica immagine di Agrigento medievale è un disegno anonimo del 1584, conservato nella Biblioteca Angelica di Roma; esso mostra il circuito delle mura entro le quali, tra le stradine anguste e tortuose di derivazione araba, si sviluppa l'agglomerato urbano (Fig. 1). Nella parte più alta e antica del nucleo urbano medievale, denominato Terra Vecchia, separato dalla città bassa da un vallone, emergono i più importanti edifici noti come lo Steri dei Chiaromonte, la Cattedrale, intitolata a San Gerlando, il Palazzo vescovile, il complesso di Santo Spirito, il castello regio e la chiesa di Santa Maria dei Greci. I borghi duecenteschi di San Francesco, San Michele e San Pietro, che si trovavano fuori le mura nella parte più bassa della città chiamata Terra Nuova, furono inglobati nella nuova cinta muraria, restaurata nel XIV secolo. La fisionomia della città dell'epoca è da ricondurre principalmente alla presenza della famiglia feudale dei Chiaramonte, originaria della Francia, insediatasi in Agrigento dal 1342, e rimasta al potere sino alla caduta della signoria con la morte di Andrea, ultimo discendente della stirpe, decapitato a Palermo nel 1392. Tra la fine del Duecento e l'inizio del Trecento, le chiese e i conventi assumono un ruolo fondamentale nella vita cittadina, grazie al potere esercitato dal vescovo, figura di primo piano nell'assetto politico ed economico della città medievale. L'insediamento degli ordini monastici incrementa la proliferazione di nuovi edifici conventuali e alimenta la necessità di ristrutturare le chiese esistenti, danneggiate durante gli scontri avvenuti contro i musulmani ${ }^{1}$. Numerosi sono i templi greci dell'Impero d'Oriente che vengono trasformati, di conseguenza, in chiese cristiane, secondo una consuetudine largamente promossa dalle autorità pontificie dal VI secolo d.C. ${ }^{2}$. Con l'occupazione islamica dell'829, l'antica città greca di Akragas - che per motivi difensivi si era ritirata sull'acropoli, identificata sulla collina di Girgenti ${ }^{3}$

\footnotetext{
${ }^{1}$ Sardina, 2011: 17-19.

Zarbo, 2010: 99-106.

Schubring, 1887: 72-85.
} 


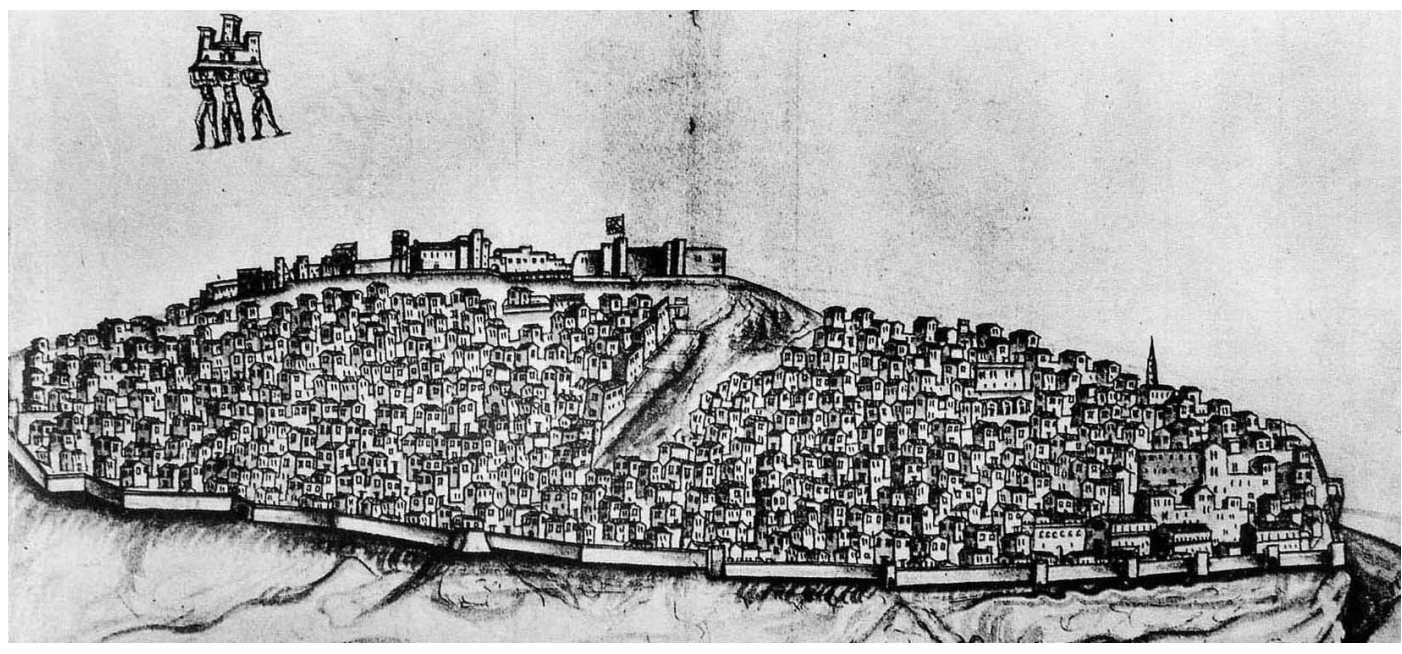

Fig. 1 Disegno di anonimo, Girgenti 1564 (Biblioteca Angelica, Roma) tratto da Liliane Dufour, Atlante storico della Sicilia, Edizioni A. Lombardi, Palermo 1992

- si espande e fortifica assumendo il nome di Kerkent. Nell'area della nuova città - così come nell'antica - non si individuano nuove costruzioni realizzate dagli arabi; pertanto, è possibile ipotizzare che i luoghi di culto cristiano abbiano continuano a svolgere la propria funzione senza aver subito modifiche sostanziali nell'assetto architettonico. Gli arabi, infatti, pur vietando la costruzione di nuove chiese, avevano consentito ai popoli sottomessi di continuare a professare la propria religione ${ }^{4}$. Il tempio della Concordia - trovandosi extra moenia rispetto al nuovo nucleo urbano - è un chiaro esempio di trasformazione di un edificio pagano in basilica cimiteriale cristiana, realizzata alla fine del VII secolo d.C. per opera del vescovo Gregorio, che la intitolò ai santi Pietro e Paolo 5 .

Alla fine del XIII secolo anche il tempio dorico sottostante la chiesa - di probabile origine bizantina $^{6}$ - di Santa Maria dei Greci, situato sulla collina di Girgenti, subisce alcuni trasformazioni per essere utilizzata durante la dominazione araba come chiesa cristiana di culto greco. La chiesa, costruita sul tempio in rovina, venne adattata alla nuova funzione con i materiali superstiti del tempio stesso, secondo una consuetudine dal forte significato simbolico ${ }^{7}$.

Negli anni successivi, la struttura architettonica originaria ha subito notevoli interventi di restauro e lavori di completamento di carattere ornamentale, che hanno determinato la sua trasformazione in quella che è la sua conformazione attuale ${ }^{8}$.

\section{Primo intervento di restauro. Periodo normanno-svevo-chiaramontano. 1087-1392.}

Nell'anno 1086 Ruggero il Normanno conquista la città di Kerkent, rinominata Girgenti, sottraendola dopo 259 anni al dominio arabo. Il sovrano organizza uno stato compatto e potente, il cui simbolo è rappresentato dal Castello dell'Itria, realizzato dentro le mura della città. La cittadella normanna aveva una superficie complessiva di 22 ettari e presentava un assetto viario sinuoso, con strade strette e cieche ${ }^{9}$. In questi anni, le fortificazioni esistenti sono potenziate ed estese verso sud-

\footnotetext{
${ }^{4}$ Bosco, 1973: 22-32.

${ }^{5}$ De Miro, 1994: 47. Mercurelli, 1948: 26-43.

${ }^{6}$ Vicari, 2005: 4.

7 Zarbo, op. cit.: 245.

${ }^{8}$ Revenga, 2012, 91-93

9 Di Giovanni, 1997: 3-9.
} 


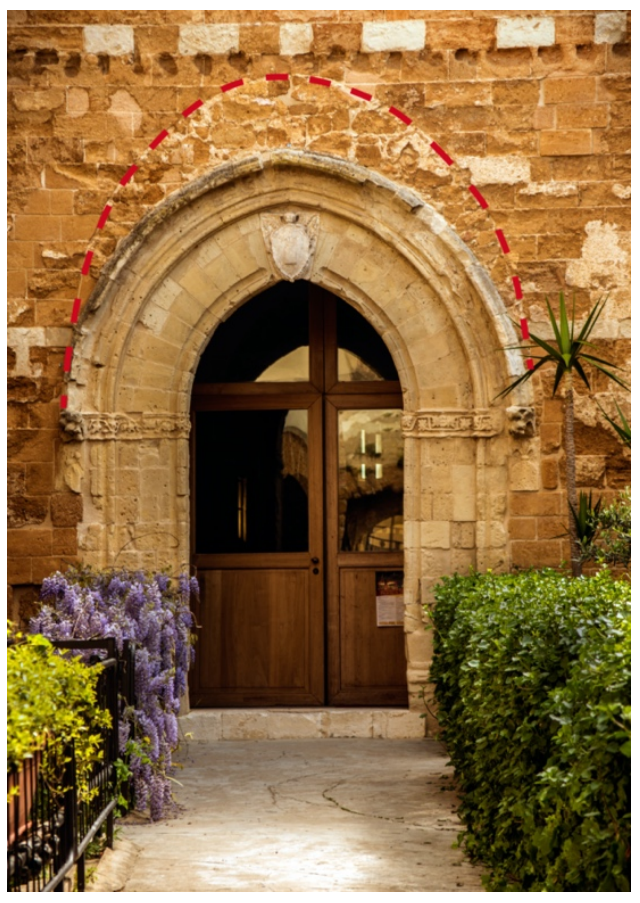

Fig. 2 Particolare del portale d'ingresso della chiesa di Santa Maria dei Greci. Nella foto a destra è evidenziato con un tratteggio in rosso l'arco preesistente (foto di R. Magrì)

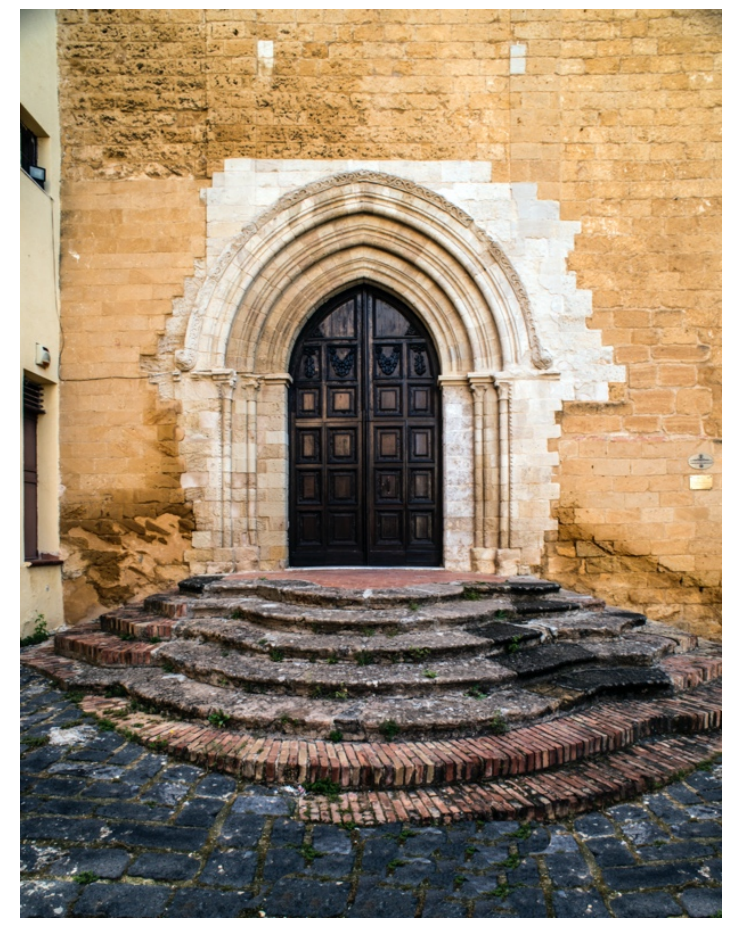

Fig. 3 Particolare del portale d'ingresso del Monastero di Santo Spirito (foto di R. Magrì)

est racchiudendo al loro interno la Terra Nuova; il quartiere arabo del Rabato (Rabhad) manteneva così una posizione periferica, contrapposta alla parte di città situata sulla collina - denominata Hisn - dove furono edificati i più importanti civili e religiosi ${ }^{10}$.

Con il decreto del 1093, Ruggero nomina Gerlando di Besancon, suo cugino, a vescovo della città; egli, tra il 1093 e il 1099, fece edificare sulla collina di Girgenti il primo impianto della nuova cattedrale e l'Episcopio, e incominciò la ricostruzione del castello demolito dai musulmani ${ }^{11}$. Il vescovo Gerlando, come riportato nel Libellus de successione pontificum Agrigenti ${ }^{12}$ edificò nell'arco di sei anni episcopium et curiam prope castellum per timore degli attacchi dei Saraceni e il 4 aprile del 1099 consacrò la Cattedrale, dedicandola a Santa Maria e all'apostolo Giacomo ${ }^{13}$. Durante i lavori di costruzione, la preesistente chiesa di Santa Maria dei Greci viene utilizzata come cattedrale, e pertanto fu considerata come "prima cattedrale di Agrigento", già in uso quindi da cinque secoli e sopravvissuta alla dominazione araba ${ }^{14}$. Nel primo periodo della dominazione normanna in Sicilia la maggior parte delle chiese edificate sugli avanzi dei templi greci, ad eccezione della cattedrale di Siracusa, subisce per lo più interventi di rifacimento di portali e soffitti, unitamente ad interventi di manutenzione che certamente hanno garantito nel tempo la conservazione delle fabbriche originarie. Anche Santa Maria dei Greci, probabilmente per opera del vescovo Gerlando, o dei successori,

${ }^{10}$ Il quartiere del Rabato (dall'arabo rabadh, sobborgo) si trova nell'estremità occidentale della Collina di Girgenti,. Alcuni quartieri sorti extra moenia nel periodo normanno e federiciano, chiamati Rabati, vennero poi inclusi all'interno delle fortificazioni nel XIII secolo, mentre il Rabato o Rabatello rimase all'esterno e fu densamente popolato sino alla frana di Agrigento del 1966. Al suo interno, oltre alle grotte trogloditiche - case scavate nella roccia in epoca protostorica nella zona denominata "Balatizzo"- si trovano importanti edifici come il complesso di Santa Croce, la chiesa di Santa Caterina e le Fabbriche del Carmine, del periodo chiaramontano. Miccichè, 2006: 30-33.

${ }^{11}$ Di Franco, 2016: 19-20.

12 Si tratta di un codice pergamenaceo compilato tra il 1250 e il 1260 per volere del vescovo Raynaldo Acquaviva, contenente una lista dei possedimenti della chiesa di Agrigento. Picone, 1866: 454.

13 Sardina, op. cit.: 31.

14 De Gregorio, 1996: 43. 

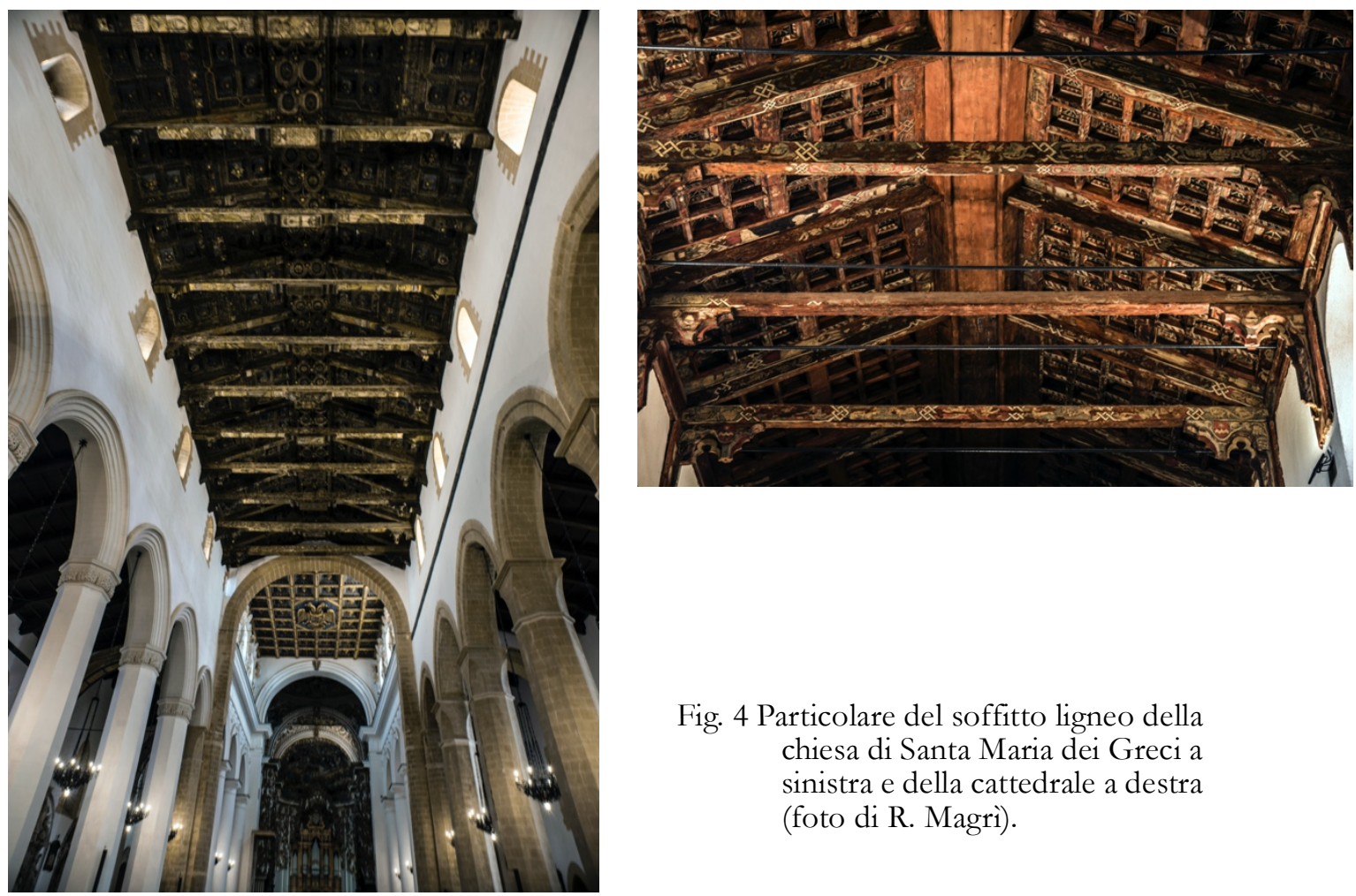

Fig. 4 Particolare del soffitto ligneo della chiesa di Santa Maria dei Greci a sinistra e della cattedrale a destra (foto di R. Magri).

subisce alcune modifiche che presentano i caratteri stilistici propri dell'architettura del periodo normanno-svevo. Con l'arrivo in città del re Federico II di Svezia nel 1232 si assiste ad un progressivo cambiamento della fisionomia della società agrigentina e di conseguenza della sua struttura urbana. Intorno al 1340 le famiglie nobiliari più influenti, come quella dei Chiaramonte, assumono un ruolo di primo piano nella gestione dell'amministrazione cittadina. Per accentuare il proprio prestigio la città è abbellita con ricchi palazzi e imponenti chiese e monasteri, realizzati dentro e fuori le mura, secondo lo stile definito chiaramontano, contrassegnato da forme, elementi decorativi e geometrie appariscenti, desunti dall'architettura gotica-catalana, con una connotazione del tutto "isolana". In tal senso, attraverso lo studio dei portali di ingresso degli edifici della Sicilia occidentale è stato possibile definire i caratteri specifici dello stile e valutare le affinità e/o le differenze tra i diversi esempi $^{15}$. Il portale si configura infatti come un elemento architettonico di grande rilevanza nei complessi medievali ed è caratterizzato dalla ripetizione di archi a sesto acuto sottolineati da eleganti cornici in pietra squadrata e da esili colonnine che scendono dall'imposta degli archi verso il pavimento. In particolare, nel caso dello stile chiaramontano, queste caratteristiche sono riscontrabili negli ornamenti che accomunano gli edifici, molti dei quali furono commissionati dalla Marchisia Prefolio $^{16}$, che fece realizzare in città le maggiori architetture religiose, come il monastero di Santo

\footnotetext{
${ }^{15}$ Inzerillo, 2004: 35-36.

${ }^{16}$ La marchesa Rosalia Prefoglio, insieme al marito Federico I, furono esponenti della famiglia Chiaramonte di grande importanza nella storia della città di Girgenti. Dal loro matrimonio nacquero i tre figli Manfredi, Giovanni I e Federico II, che resero grandiosa la famiglia, diventando i principali promotori della rinascita culturale che coinvolse molte città della Sicilia del XIV secolo. Le spoglie della marchesa si conservano nella cattedrale di Agrigento, dove i figli fecero costruire un imponente sepolcro. Di Giovanni, op. cit.: 176-177.
} 


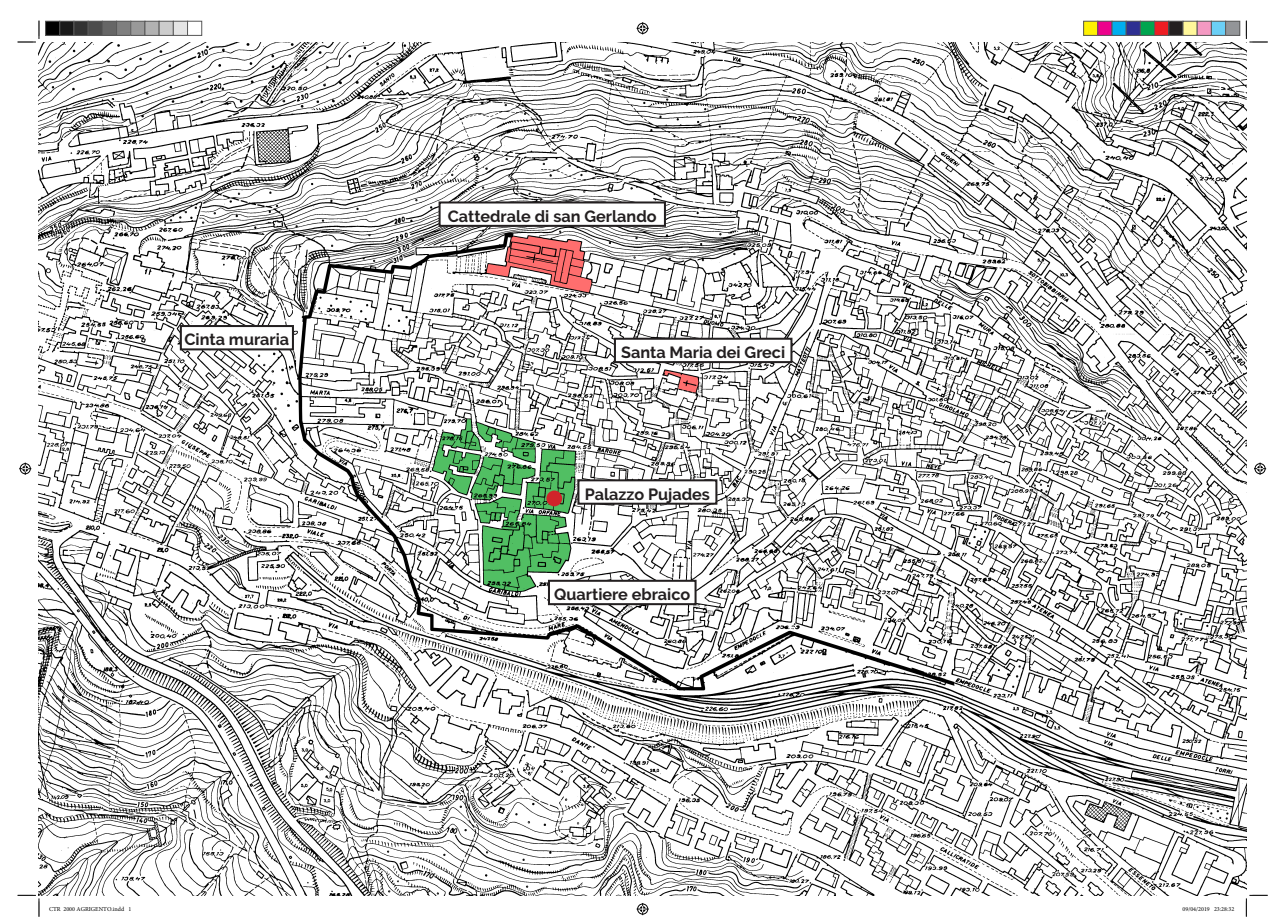

Fig. 5 Planimetria del centro storico con l'individuazione del quartiere ebraico (elaborazione grafica a cura di R. Magri).

Spirito $^{17}$ e la chiesa e il convento di San Francesco d'Assisi ${ }^{18}$. Il portale di accesso alla chiesa di Santa Maria dei Greci mostra evidenti analogie con i portali della chiesa madre di Naro e di Caltabellotta, e del castello di Favara, risalenti al periodo chiaramontano. Realizzato in mattoni squadrati, con una successione di archi di elegante fattura, spicca dalla muratura color ocra e si contraddistingue come l'elemento più significativo del prospetto della chiesa. La compagine muraria mostra la presenza di un preesistente arco a sesto acuto, con una curvatura di altezza maggiore, probabilmente risalente al periodo bizantino, segnale di una prima trasformazione architettonica dell'edificio (Fig. 2).

"L'interno della chiesa è stato più volte rimaneggiato. Il portale non risale al primo momento della trasformazione, ma fu preceduto almeno da un altro più archiarcuato, il cui svolgimento è suggerito da una linea che si coglie nei conci di riempimento precedente e nuovo",19.

Alcune affinità sono riscontrabili, inoltre, seppur in modo semplificato, tra il portale di Santa Maria dei Greci e quello del refettorio della badia di Santo Spirito, in quanto entrambi presentano cornici multiple intagliate a forte rilievo, fasce piane in pietra squadrata, proporzione delle forme e una profonda strombatura su piani degradanti. La differenza tra i due portali sta nella presenza in

${ }^{17}$ La Chiesa di Santo Spirito, nota come Badia Grande (Bataranni), insieme al monastero costituisce uno dei monumenti più importanti di Agrigento. Le notizie storiche sulla sua fondazione sono molto lacunose: certa è la sua presenza alla fine del 1300, quando la marchesa Prefoglio, dopo avere fondato il monastero delle Vergini di Santo Spirito, la dona alla confraternita dei cistercensi. Sorto con probabilità sui resti dell'antico palazzo della famiglia Chiaramonte poi costruito nuovamente sui luoghi dell'attuale Seminario Vescovile - non mostra più i suoi caratteri originari, a seguito dei numerosi interventi di restauro realizzati nel corso degli ultimi tre secoli. La chiesa e il monastero costituiscono un unico comparto edilizio prospiciente la piazzetta delimitata da altre costruzioni. La facciata principale dell'edificio mostra elementi riconducibili al periodo chiaramontano, come il portale e il rosone, mentre altri elementi, come la cella campanaria, sono di epoca barocca. Di Giovanni, op. cit.: 236-238.

${ }_{18}$ La chiesa di San Francesco fu costruita da Federico II, terzogenito della marchesa Prefoglio e completata nel 1307. Addossati alla basilica, intitolata all'Immacolata, si trovano i resti del convento chiaramontano di San Francesco d'Assisi, caratterizzato da un prezioso portale a sesto acuto affiancato da due bifore. La compresenza di elementi decorativi locali con altri di matrice gotica appare in questo edificio ben proporzionata, definendo un insieme pregevole e di rara eleganza. Inzerillo, op. cit.: 48 .

Peri, 1962: 80. 

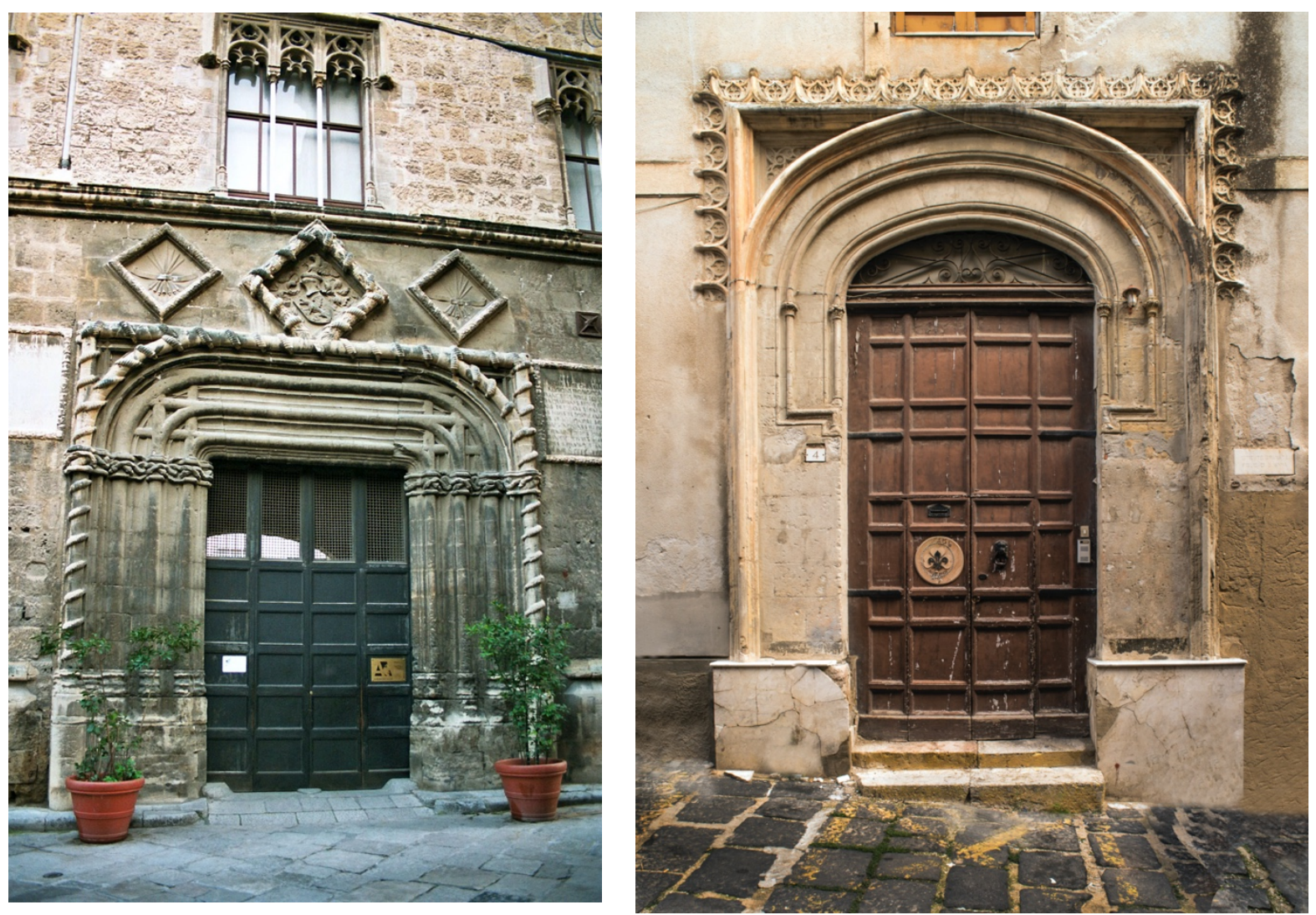

Fig. 6 Confronto tra i portali di palazzo Pujades ad Agrigento e di palazzo Abatellis a Palermo (foto di R. Magri).

Santo Spirito di una coppia di snelle colonnine, poste ai lati, sormontate da un piccolo capitello (Fig. 3).

Nel lungo arco temporale della dominazione normanno-svevo-angioina, la chiesa dunque sostanzialmente subisce solo alcune parziali trasformazioni, una delle quali ha interessato tra l'altro il soffitto ligneo a capriate dipinte, oggetto di interventi di manutenzione ${ }^{20}$ (Fig. 4).

\section{Secondo intervento di restauro. Periodo spagnolo. 1392-1720.}

Con la fine dei Chiaromonte, Girgenti - acquisita al demanio regio dopo lo sbarco in Sicilia del re Martino I d'Aragona avvenuto nel 1392 - rinnovò il proprio patriziato urbano, accogliendo al suo interno nuove personalità fedeli alla corona, dotati dal re di proprietà, titoli e privilegi. Tra questi, assunsero un ruolo di primo piano gli esponenti dell'antica comunità ebraica della città, la Aljama, una delle più consistenti dell'isola durante la prima metà del Quattrocento. La sua affermazione è favorita dall'avvento di nuove ondate migratorie e dalla protezione vicereale, la quale sostenne, in particolare, la fortunata ascesa della nobile famiglia di origine spagnola dei Pujades. Il quartiere ebraico di Girgenti - la Giudaica - ubicato fra la chiesa di san Domenico e quella di san Giacomo, inizialmente, come il vicino Rabato, era collocato fuori le mura; in seguito, con l'ampliamento delle fortificazioni, avvenuto nel corso del XIV secolo, venne inglobato nella città (Fig. 5). La comunità svolgeva un'intensa e ricca attività commerciale, che coinvolgeva ogni aspetto della vita cittadina, creando i presupposti per lo sviluppo di un'intensa attività edilizia. Sorgono, infatti, i più importanti edifici e palazzi nobiliari, situati nella parte bassa della città, come il palazzo Montaperto, il palazzo

\footnotetext{
${ }^{20}$ Vicari, op. cit.: 11.
} 

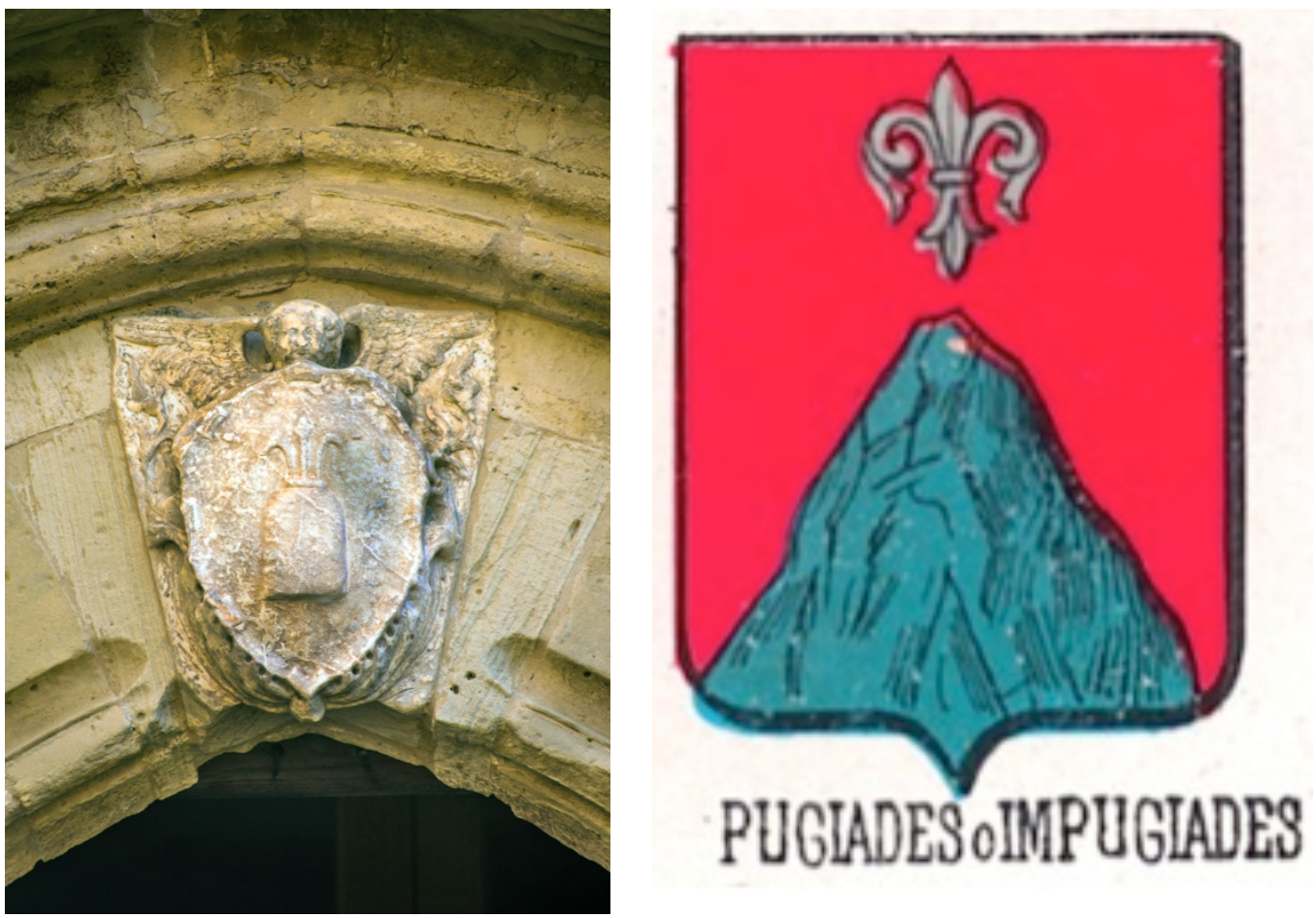

Fig. 7 Lo stemma della famiglia Pujades o Pugiades o Impugiades, raffigurante un monticello coronato sulla vetta da un giglio trilobato d'argento (da Nobiliario di Sicilia del Dott. Antonino Mango di Casalgerardo), raffrontato allo stemma in pietra scolpita posto sulla chiave di volta dell'arco del portale (foto di R. Magrì).

De Marinis, la chiesa del SS. Salvatore e la principale istituzione della comunità ebraica - la sinagoga - detta anche Meskita. All'interno del quartiere sorgeva il palazzo Pujades - attuale Istituto Granata -, i cui proprietari erano ricchi mercanti ebrei provenienti da Barcellona. I discendenti della famiglia, Michele e Matteo Pujades, vissuti negli anni a cavallo tra il 1400 e il 1500, erano ben amalgamati nell'ambiente nobiliare locale e si contraddistinsero per rilevanti iniziative nell'ambito cittadino. Il ruolo dei Pujades, dunque, è di primo piano nella storia politica ed economica della città quattrocentesca e il loro prestigio si manifesta attraverso la realizzazione di sontuosi palazzi destinati alla propria dimora, ma anche con interventi rivolti alla comunità. Il palazzo nobiliare della famiglia presentava affinità evidenti con il palazzo di Gaspare De Marinis, barone di Muxaro (oggi non più esistente), attribuito al maestro Matteo Carnilivari ${ }^{21}$ - con molta probabilità operativo ad Agrigento antecedentemente al 1487 - situato "in contrada del Portulano", zona compresa tra via Orfane, vicolo Teatro, piano Barone e piazza Municipio, corrispondente alla parte bassa del quartiere Terra Vecchia. Da un atto stilato nel 1605 dal notaio Bartuglia di Girgenti si apprende in primo luogo che il palazzo De Marinis sia stato donato dai proprietari al Clero della cattedrale e in secondo che la sua posizione sia stata prospiciente al Piano Barone, ossia nella parte bassa della Terra Vecchia. Si deduce, inoltre, nel palazzo la presenza di un portale maggiore molto simile a quello del palazzo

\footnotetext{
${ }^{21}$ Carnilivari Matteo, architetto nato a Noto (SR) nella prima metà del XV sec, considerato uno dei maggiori esponenti del '400 siciliano. Dopo aver lavorato nella sua città natale e in altre località della Sicilia, nel 1487 si trasferisce a Palermo, a servizio del banchiere e mercante di origine pisana Guglielmo Aiutamicristo, per il quale esegue il restauro ed ampliamento del castello chiaramontano di Misilmeri. Opera principale dell'architetto è da considerarsi il palazzo Abatellis a Palermo, costruito con Nicolò Grisafi ed altri maestri intorno al 1491, in contemporanea con l'esecuzione della grande dimora degli Aiutamicristo. È attribuito al Carnalivari anche la celebre chiesa della Catena a Palermo. La sua produzione diventa, dunque, un unicum tale da caratterizzare l'architettura di molti centri della Sicilia, ponendosi tra il nuovo stile di derivazione tardogotica, con chiare influenze di origine catalana, e la tradizione angioina e aragonese, fortemente presente sul territorio. Sutera, 2006: 89-94.
} 


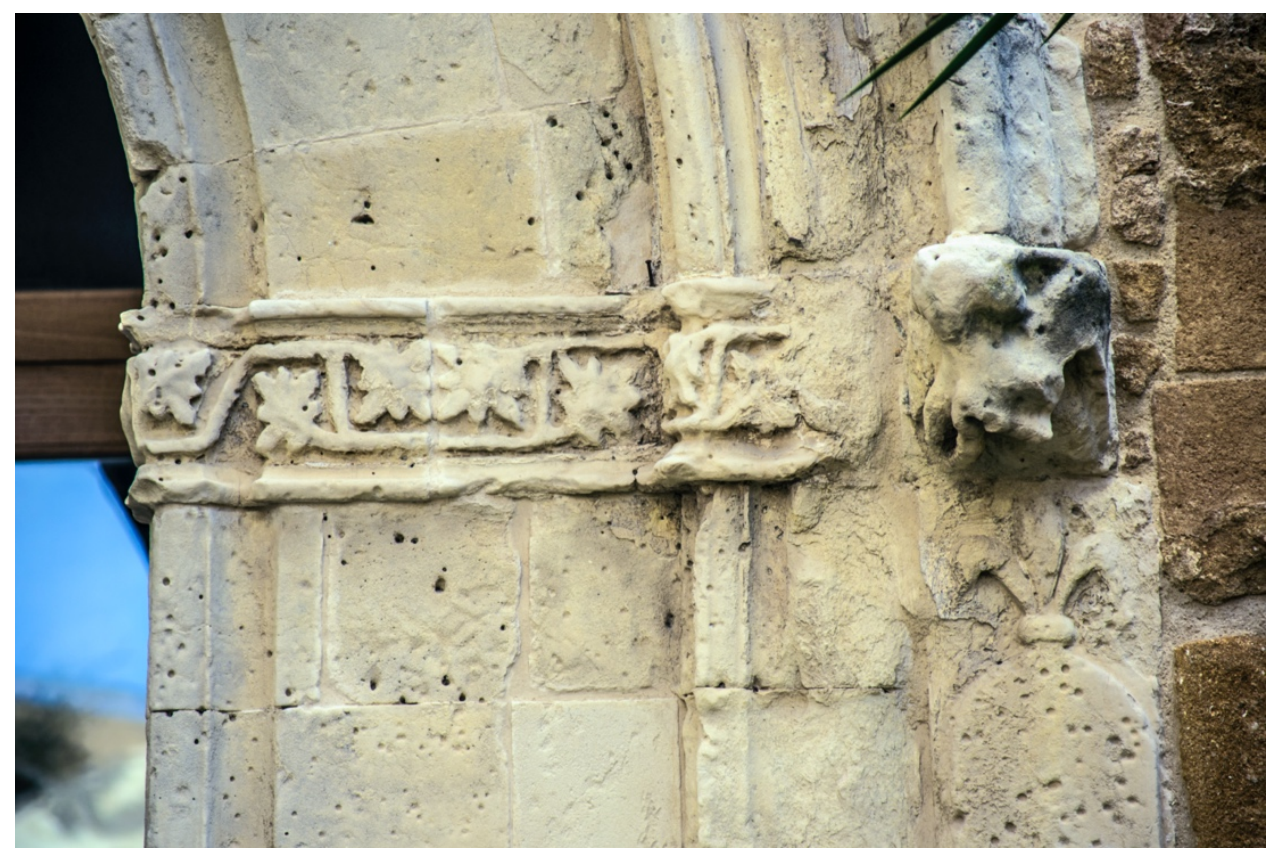

Fig. 8 Particolare della fascia decorativa orizzontale del portale (foto di R. Magrì).

Abatellis di Palermo ${ }^{22}$. Per il palazzo Pujades non si hanno notizie certe, ma alcuni riscontri ci permettono di ipotizzare che l'architetto della fabbrica sia stato lo stesso Carnilivari ${ }^{23}$. Il palazzo, ubicato anch'esso nella "contrada del Portulano", secondo un documento del 1493, era "noviter fabricatas per $n$. Matteum Pujades, eam contingentes parietem dicte Judaice, juxta fabricationem ipsarum domorum per ipsum n. Matteum $[\ldots]^{24}$, quindi all'interno del quartiere della Giudecca, adiacente alla Mesquita, coerentemente al fatto che il ramo agrigentino dei Pujades sia stato di religione ebraica. Il palazzo della famiglia, costruito nel 1492, mantiene la sua conformazione fino al 1878, quando divenne la sede di un istituto religioso. L'arco a sesto ribassato policentrico del portale mostra tre cornici sporgenti; la più esterna continua il suo andamento formando i piedritti dell'arco, mentre la seconda si interrompe ad angolo retto poco al di sotto del piano di imposta dell'arco, per poi ricongiungersi alla terza. Questa particolare conformazione di arco si riscontra in alcuni edifici eseguiti dal Carnilivari - seppure con particolari di diversa tipologia - nella Sicilia occidentale, come il palazzo Abatellis a Palermo (Fig. 6). Nelle sue forme, l'architetto realizza la sintesi tra il gotico-aragonese e il primo rinascimento, proponendo un portale definito da cornici con andamenti rettilinei e sapienti incroci, unico nel suo genere. La decorazione leggera, presente nei capitelli delle colonnine, si contrappone al vigoroso cordolo che incornicia l'arco esterno del portale, sormontato da tre riquadri in diagonale, uno dei quali al centro con lo stemma di famiglia ${ }^{25}$.

Tra i lavori di restauro eseguiti tra gli ultimi decenni del ' 400 e i primi decenni del ' 500 , sotto il patrocinio dei Pujades, si annoverano [... le opere di abbellimento che interessarono la chiesa di Santa Maria dei Greci: il portale con lo stemma della famiglia, gli affreschi interni, il restauro del tetto ligneo dipinto di matrice chiaramontana. La presenza dello stemma Pujades alla base dei due capitelli pensili nell'arcata d'ingresso, oltre che di un terzo scudo di marmo inserito successivamente al culmine dell'ogiva, non lasciano dubbi sulla committenza del portale, suggerendo l'ipotesi di estendere il patrocinio del casato anche alle opere pittoriche dell'interno ${ }^{26}$. Lo stesso Illuminato Peri aveva attribuito con certezza ai Pujades il lavoro di ripristino strutturale del portale

\footnotetext{
22 Alajmo, 1962: 20.

23 Alajmo, op. cit.: 21-25. Sutera, 2006: 90.

24 Zalapì, 2007: 388-389.

25 Inzerillo, op. cit.: 75-76.

${ }^{26}$ Zalapì , op. cit.: 387.
} 
Fig. 9 Ciclo di affreschi dedicati alla Vergine Maria sulla parete della navata laterale destra (foto di R. Magri).

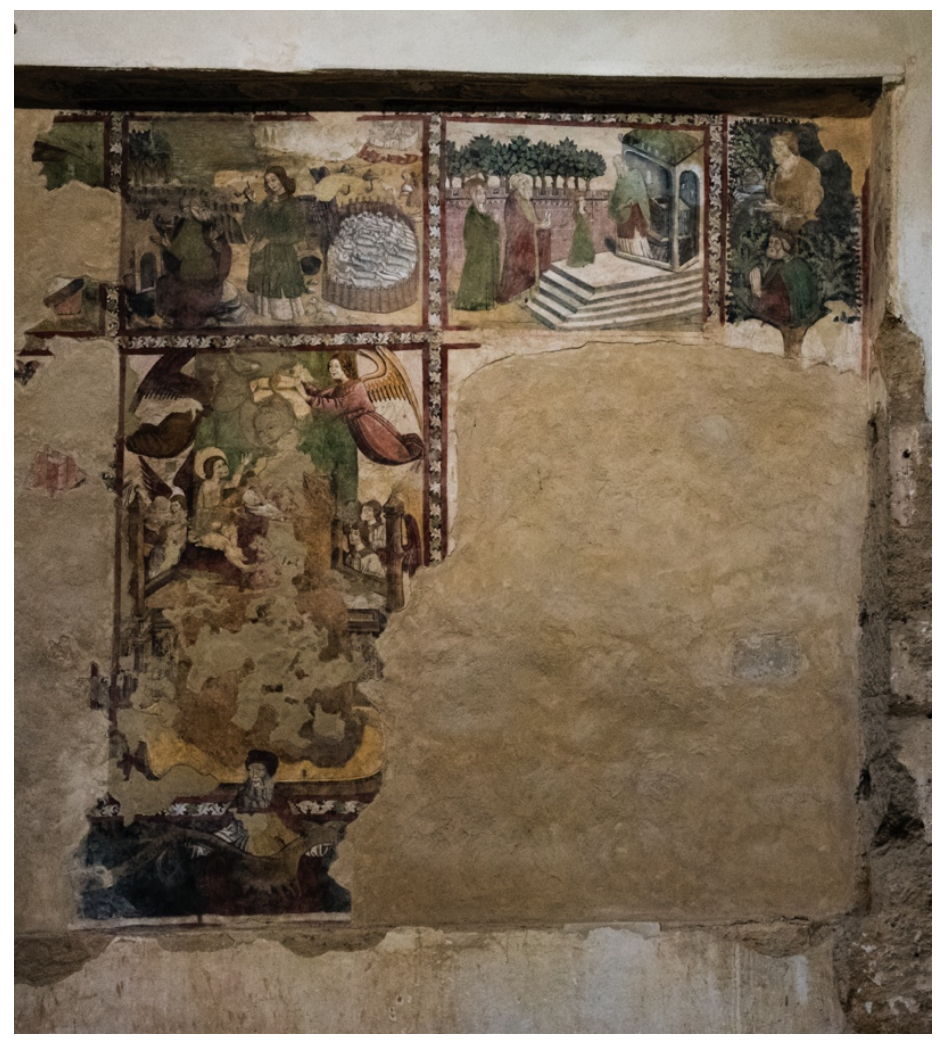

della chiesa, proprio per la presenza sulla sommità del portale dello stemma di famiglia: [...] I Pujades vi posero lo stemma, togliendone (e l'operażione è pure evidente) un paio di conci al centro ${ }^{27}$ (Fig. 7). Altro elemento significativo è la fascia orizzontale che delimita la parte superiore del portale ai piedritti dell'arco, ornata con modanature realizzate a motivi floreali, riscontrabile in molti portali eseguiti dal Carnilivari e dalla sua scuola (Fig. 8). La famiglia Pujades scelse di patrocinare i lavori di abbellimento proprio della chiesa di Santa Maria dei Greci per esaltarne il valore spirituale, essendo stata la prima cattedrale di Girgenti, e di conseguenza acquisire prestigio nei confronti del clero. La chiesa, inoltre, era in prossimità del palazzo padronale in quello stesso pianoro, dove si affacciavano i più importanti edifici della città dell'epoca. Elemento di non secondaria importanza, è poi la conversione alla religione cattolica di Gianmatteo Pujades - identificabile con Matteo junior ${ }^{28}$ - certificata da un atto notarile del 1493, il quale manifesta con questo gesto di dedizione nei confronti della chiesa cattolica l'effettivo cambiamento di fede. Molteplici sono i fattori che ci inducono ad attribuire i lavori di abbellimento della chiesa di Santa Maria dei Greci a Matteo Carnilivari. In primo luogo è da citare un contratto stilato nel 1499 dal fabricator Antioco de Cara, artista-scultore collaboratore del maestro, con il quale egli si impegnava a recarsi in Agrigento per "laborare, facere, murare et intaglare totam illam quantitatem maragmantis", su richiesta di Matteo Pujades e per un imprecisato cantiere ${ }^{29}$. Inoltre, un altro elemento che supporta la nostra ipotesi è il rapporto di amicizia tra Matteo Pujades e Francesco Abatellis - entrambi portolani $i^{30}$ uno in Agrigento e l'altro a Palermo -, per i quali il maestro netino avrebbe eseguito sia il palazzo commissionato dalla nobile famiglia palermitana che il palazzo dei Pujades. Tra i lavori di "abbellimento" fu realizzato un nuovo sistema di copertura a capriate dipinte, in sostituzione del preesistente. Le caratteristiche stilistiche ed architettoniche della copertura della chiesa di Santa Maria dei Greci sono riconducibili per affinità al soffitto ligneo della cattedrale di san Gerlando, realizzato nel XVI secolo dal vescovo Giuliano Cybo. Le varie fasi di costruzione della

\footnotetext{
${ }^{27}$ Peri, op. cit.: 80.

${ }_{28}$ Picone, op. cit.: 642, n.4

${ }_{30}^{29}$ Zalapì, op.cit.: 389. Di Fede, 2006: 69.

30 Il maestro portulano, figura esistente dal XII sec. fino al 1816, era una grande autorità del regno, che sovrintendeva alle marine e ai porti autorizzati ad esportare i cereali (caricatori).
} 

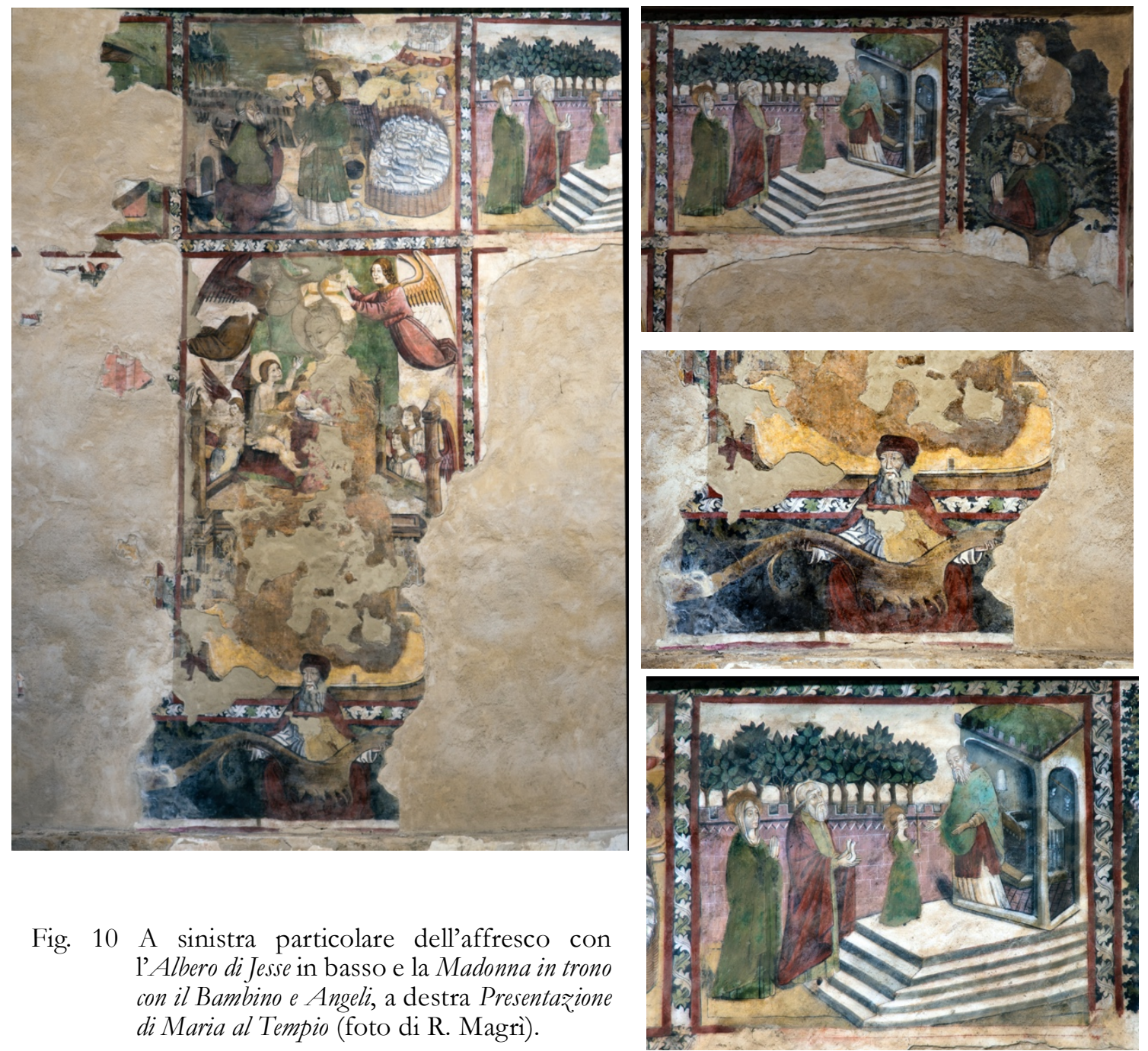

basilica hanno creato una sovrapposizione di stili, evidente anche nel sistema della copertura. L'impianto originario, risalente al periodo chiaramontano, è suddiviso in tre campate realizzate con un soffitto cassettonato a lacunari dorati, la cui decorazione è stata attribuita ad un pittore di scuola spagnola; le capriate lignee a vista sono state decorate con figure di santi tra il 1511 e il 1514 da Masolino da Floregia da Girgenti. Negli ultimi anni del '500 Carlo V fece arricchire la campata centrale con 48 cassettoni dorati, al centro dei quali spicca l'aquila dorata bicefala, stemma degli Asburgo; l'ultimazione dei lavori, con i cassettoni a vista dei bracci laterali, venne realizzata nel 1663 dal vescovo Gisulfo ${ }^{31}$. La stessa tipologia costruttiva si riscontra nella copertura di Santa Maria dei Greci realizzate a due falde cassettonate dipinte, sorrette da capriate decorate con immagini sacre e motivi ornamentali attribuiti al pittore Innocenzo Mascarella, autore tra l'altro degli affreschi della chiesa di San Nicola ${ }^{32}$.

Nella storia dei restauri dell'edificio si annovera la realizzazione del ciclo di affreschi posti all'interno della chiesa sulla parete della navata destra, raffigurante le Storie dellinfanzia della Vergine (1490-1510). L'opera, pur non essendo un intervento di restauro, è considerata un'opera di abbellimento

\footnotetext{
${ }^{31}$ La prima edificazione della Cattedrale risale al periodo normanno (1096-1102) in un luogo fortificato sulla collina di Girgenti, tanto da essere denominata "ecclesia munita", ossia luogo di culto e di difesa. Il fronte principale, preceduto da un'ampia scalinata, è caratterizzato dalla chiesa e dall'imponente torre campanaria. Di notevole pregio architettonico è il portale di epoca barocca e il soprastante rosone. L'interno è a tre navate suddiviso da due file di colonne a base ottagonale e circolare, sormontate da archi a sesto acuto e a tutto sesto. Di Giovanni, op. cit.: 269-271.

Zalapì, op.cit.: 393 n.22.
} 

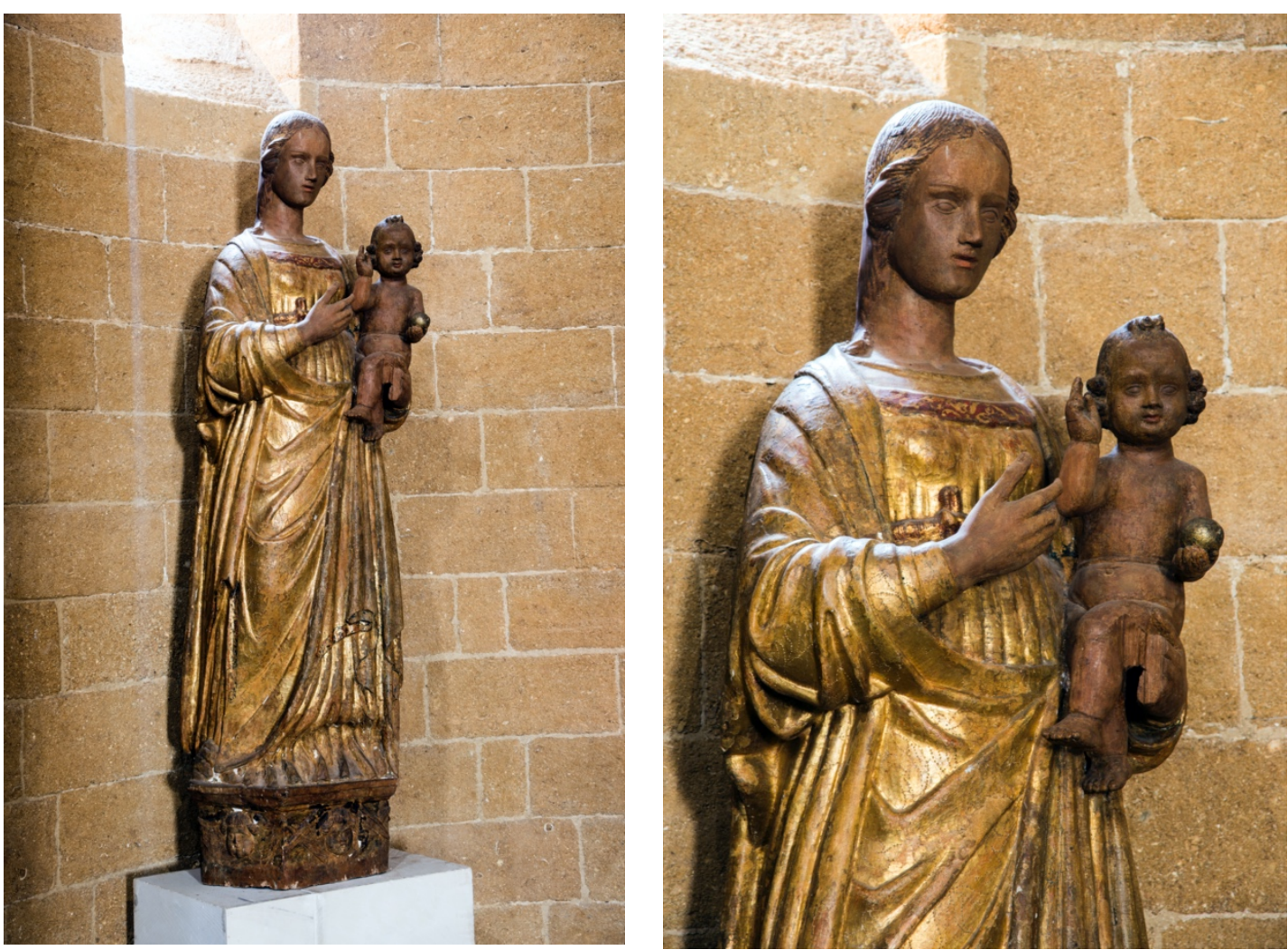

Fig. 11 Sarcofago in marmo, crocifisso ligneo e Madonna con il Bambino (foto di R. Magrì).

della chiesa (Fig. 9). Il ciclo è attribuito da alcuni studiosi allo stesso Mascarella, mentre per altri è riferibile a un maestro anonimo siciliano di grande abilità, ma ancorato alle descrizioni pittoriche di tipo tardomedievali, lontane dagli schemi prospettici rinascimentali. La struttura dell'affresco, inserito in un incasso della parete, è suddivisa in scomparti all'interno dei quali si svolgono le scene legate alla vita della Vergine Maria. I riferimenti alla cultura ebraica rappresentati nei riquadri portano ad attribuire la commissione dell'opera alla stessa famiglia Pujades, come già detto, di origini giudee, che aveva voluto fortemente legare il proprio nome a quello della piccola ma preziosa chiesa cittadina. Il pittore mostra, infatti, di conoscere usi e costumi ebraici, riprodotti con dettagli all'interno delle scene. La figura biblica di Jesse, padre della discendenza davidica, raffigurata nel riquadro in basso intitolato l'Albero di Jesse, rappresenta l'origine dell'opera, poiché dalle sue mani che reggono una radice si dirama simbolicamente l'albero genealogico con gli antenati di Maria e di Gesù Cristo. Nell'angolo in alto a destra sono rappresentate due figure - una delle quali con una corona regale - che emergono dalle foglie dell'albero. L'affresco mostra al centro la Madonna in trono con il Bambino e Angeli, mentre per i comparti laterali - non più esistenti - si possono ipotizzare altre scene legate all'intero ciclo dedicato alle Storie; nei tre riquadri superiori, rimarcati dalla cornice a motivi floreali, simile a quella presente sui soffitti lignei di palazzo Ajutamicristo, sono rappresentati altri episodi - L'Angelo che annuncia a Gioacchino la nascita di Maria, la Presentazione di Maria al Tempio - che compongono la storia della vita di Maria (Fig. 10).

I riquadri andati perduti, probabilmente cinque, dovevano completare la narrazione descritta nei vangeli apocrifi, comunemente conosciuti nel XIII secolo, con gli episodi dedicati all'Annunciazione, alla Cacciata di Gioacchino dal Tempio e allo Sposalizio della Vergine $e^{33}$.

\footnotetext{
${ }^{33}$ Zalapì , op. cit. 386. Vicari, op. cit.: 14.
} 

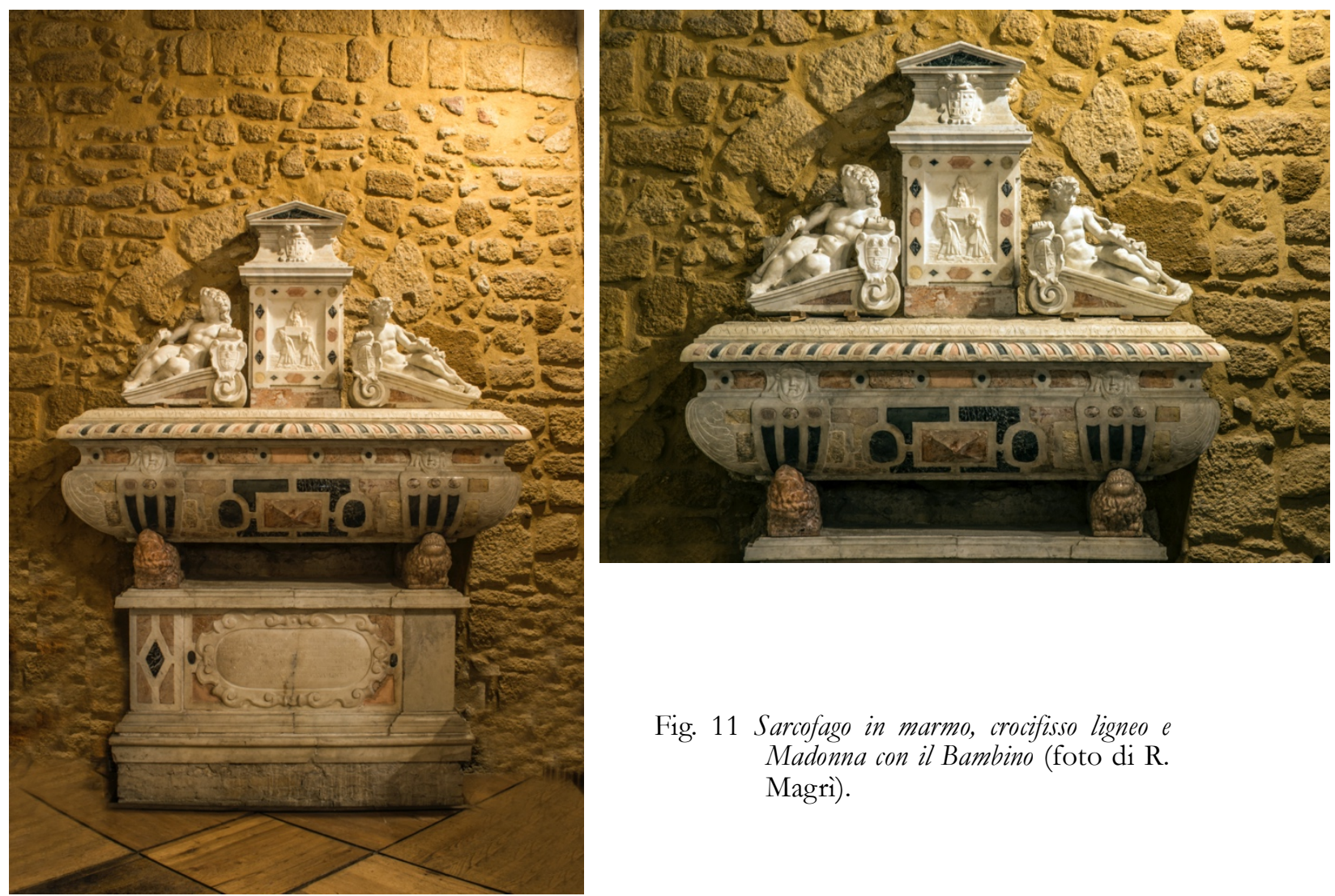

Nel 1570 la chiesa viene arricchita dalla collocazione di un monumento sepolcrale - situato in fondo alla navata destra - decorato con tarsie marmoree, contenente le spoglie dei nobili palermitani Isabella Termini e Bartolomeo Caputo o del loro figliolo. Il sarcofago, di elegante fattura, è impreziosito da due statue raffiguranti angeli e da un bassorilievo dedicato alla Madonna dell'Itria, patrona dei siciliani. Dello stesso periodo sono il crocifisso ligneo, posto sopra l'altare maggiore all'interno dell'abside centrale - di autore ignoto - proveniente dalla Cattedrale, e la statua lignea della Madonna con il Bambino (Fig. 11).

\section{Terzo intervento di restauro. Periodo austriaco-borbonico. 1720-1860.}

Dopo la guerra di successione spagnola nel 1713 il regno di Sicilia viene ceduto a Vittorio Amedeo di Savoia, il quale, imponendo il pagamento di tasse elevatissime, rese particolarmente difficile la situazione dell'isola. Nel 1720 gli Asburgo ricevono l'isola dai Savoia, in cambio della Sardegna; l'Austria, sconfitta durante la guerra di successione polacca, nel 1734 si ritira prima da Napoli e poi dalla Sicilia, che nel 1735 passa nelle mani di Carlo III di Borbone, divenuto re di Sicilia $^{34}$. Nel 1815 il congresso di Vienna riconosce a Ferdinando I di Borbone il titolo di re delle Due Sicilie. Durante questo periodo si avvia nella città di Girgenti un processo di ampliamento e rinnovamento, che vede la realizzazione di giardini, viali, ville e teatri. Nell'ambito di questa attività si inseriscono alcune operazioni di carattere manutentivo, che interessarono vari edifici religiosi e monumentali della città. Da un atto del notaio Pietro Buscemi conservato presso l'archivio Vescovile, si apprende l'attuazione di un intervento conservativo della chiesa di Santa Maria dei Greci da parte della Confraternita omonima, già presente nella chiesa sin dal 1343. La scheda dei lavori, stilata da

\footnotetext{
${ }^{34}$ Approfittando della guerra di successione polacca, nel 1734, Carlos, l'allora duca di Parma e futuro sovrano, sconfisse gli austriaci con le truppe del padre, re Filippo V di Spagna, recuperò questi regni per la sua dinastia e fu immediatamente riconosciuto per la Francia ai sensi del Primo patto familiare, nel 1737 per lo Stato Pontificio e poi per il resto degli stati italiani. Si veda, Revenga Domínguez, 2011, pp. 499-503.
} 
Giovanni Zirretta, direttore del Museo archeologico di Agrigento tra il 1927 e il 1960, descrive nel dettaglio gli interventi eseguiti nel periodo:

\begin{abstract}
Anno 1726
Copia di un documento in possesso del sac. Sanfilippo: - "Sotto il giorno 23 agosto 1726 (Pressi il Notaro P. Buscemi) - Lorenzo Contino murifabro con i rettoriori della Contraternita di santa Maria dei Greci assumeva di eseguire alcuni lavori "Con la tratta e maestria del sudetto Contino, alzare le fabriche della sudetta chiesa, di calce e quelle parti che si richiedono secondo la d'arena in forma del disegno fatto dal fratello Simone Mancuso della Congregazione dell'Oratorio, con fare il cappellone e le due cappelle collaterali do N. Signora del SS. Crocifisso, suo figlio, della maniera del sudetto disegno prescritto con fare le lanternine per dare luce alla sudetta chiesa sopra li cubulini delli sudetto cappellone e cappelle, con fare parimenti cinque archi con li mezzi archetti e suoi pilastri di pezzi e testette secondo la proporzione del sudetto disegno e con dover finalmente imbiancare di fuori della sudetta Chiesa di calce e d'arena tutte le fabbriche nuove che s'avranno da fare cose sopra e con levare uno delli due gradini (v. scalini) che sono attualmente nella fuga delli pilastri della detta Chiesa dovendone solamente restare uno proporzionato al su detto disegno, e questo tutto di rustico incominciado dal primo di Ottobre p.v. dell'anno quinta indizione intrante 1726 e necessariamente continuare e finire, quale servizio come sopra da farsi promette e si obbliga di pagare per onze 81 e 28 tari $^{\prime \prime 35}$.
\end{abstract}

L'intervento fu dettato probabilmente da nuove esigenze stilistiche e venne affidato all' architetto Simone Mancuso, il quale progettò la copertura della zona presbiteriale con tre cupolette su base ottagonale, sormontate da un lanternino e racchiuse da tiburio. La mancanza del disegno fa ipotizzare l'intervento eseguito in questa occasione: certa è l'elevazione di setti murari, eretti probabilmente per delimitare la zona absidale, e di tre cupole - con fare il cappellone e le due cappelle collaterali. Egli esegue, inoltre, l'apertura di lanternine per illuminare la zona absidale, il rifacimento degli intonaci esterni della chiesa e dei locali annessi, e la variazione degli scalini di accesso. La collocazione di cinque archi con li mezzi archetti e suoi pilastri sembra essere riferita alle strutture per il sostegno dei nuovi elementi. Nello stesso periodo, la Confraternita di Santa Maria dei Greci fa realizzare una cripta funeraria per le sepolture dei confratelli; collocata nelle fondamenta della navata centrale in un piccolo ambiente con volta a botte, presenta un sistema di sedute in pietra che svolgevano la funzione di scolatoi (Fig. 12). Nell'ultimo restauro eseguito tra il 2002 e il 2004 è stato posizionato una pavimentazione trasparente che ha reso visibile i locali sotterranei ${ }^{36}$.

Gli interventi successivi ai lavori del 1726 riguardano solamente alcuni ritrovamenti e campagne di scavo mirate alla conoscenza di un edificio complesso e per molti aspetti misterioso, caduto nell'oblio per troppo tempo. Il rinnovato interesse nei confronti della chiesa e del tempio si rivelò dal 1752 quando lo storico Giuseppe Maria Pancrazi ${ }^{37}$ rinvenne un tratto del muro del tempio dorico che egli attribuisce a Giove Polieo. Successivamente, in una incisione del 1826, Raffaele Politi ${ }^{38}$ ricostruisce le forme del lato est della chiesa, con uno schizzo prospettico che mostra una forma molto diversa da quella attuale. Nella prima metà del secolo XIX la chiesa divenne oggetto di scavi

\footnotetext{
${ }_{36}^{35}$ Miccichè, op. cit.: 370.

${ }^{36}$ Il "Progetto di restauro statico e conservativo della chiesa e dei locali annessi sopra il tempio detto di Giove Polieo", promosso dalla Diocesi di Agrigento, hanno interessato la chiesa, la sacrestia e il caseggiato sul lato nord-est. E' stata previsto il consolidamento del cunicolo laterale del crepidoma del tempio. Nello stesso periodo è stata svolta una campagna di scavi, che ha messo in luce le strutture del basamento del tempio.

Giuseppe Maria Pancrazi nella sua opera Antichità Siciliane spiegate (1751-1752) descrive in modo sistematico per la prima volta le antichità agrigentine, soffermandosi in particolare sui templi della Valle, disegnati con grande maestria da Salvatore Ettore.

38 Raffaele Politi, attivo ad Agrigento agli inizi del 1800, architetto e archeologo, si interessa agli scavi che hanno interessato Agrigento elaborando materiale grafico e descrittivo.
} 


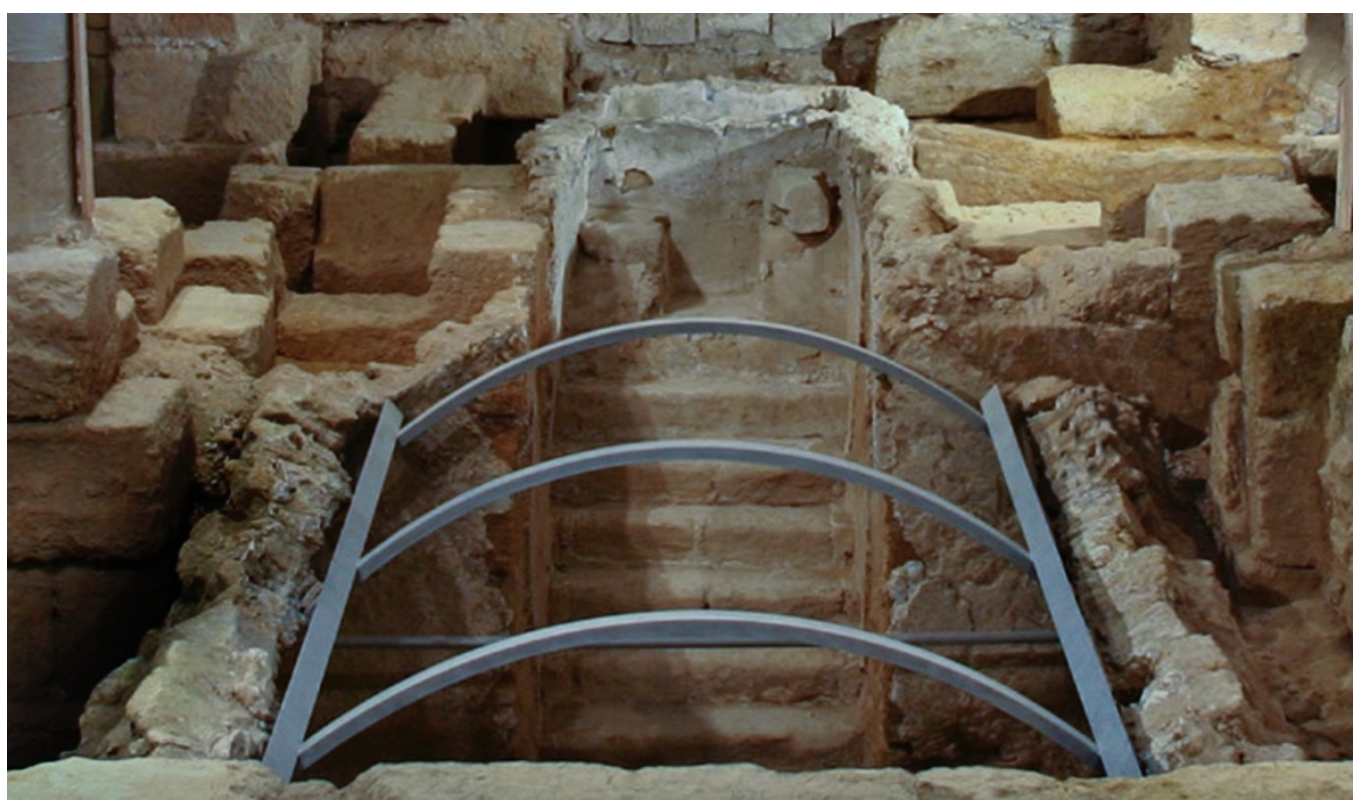

Fig. 12. La cripta funeraria di epoca settecentesca (da Vicari N., La chiesa di S. Maria dei Greci ad Agrigento, le sue trasformazioni e gli ultimi restauri, Palermo, 2005).

e ricerche, i primi dei quali furono diretti da Domenico Lo Faso duca di Serradifalco ${ }^{39}$, che mise alla luce "bastevoli avanzi" del tempio, consistenti in un lungo tratto dello stilobate settentrionale con sourastanti i tronchi dimezzati di 8 colonne [...] e in pochi elementi della trabeazione e potè così determinarne la tipologia, misurare diametri e intercolumni e larghezza complessiva, deducendone trattasi di un tempio esastilo periptero di lunghezza ancora ignota $[\ldots]^{40}$. Ulteriori studi sull'edificio - compiuti intorno al 1887 da Giulio Schubring, che per primo attribuisce il tempio ad Atena - hanno definito nel dettaglio il tempio, con " [...] le sette colonne con i tre gradini dello stilobate settentrionale rilevate dal Serradifalco [...], a cui se ne aggiungono altre due scavate più tardi ad occidente e la quarta colonna sul lato sud che si vede nella chiesa". Enumera quindi gli altri avanzi: "due perzi della trabeazione, che giacciono nel vestibolo, un piccolo tratto del muro di cella nella chiesa, brevi fondamenta del lato sud-est, alcune vestigia sul fronte occidentale $[\ldots]^{, 41}$.

Negli ultimi anni dell'Ottocento e nei primi del ' 900 si diede l'avvio agli importanti lavori di restauro, che hanno trasformato il complesso architettonico nella forma attuale.

\footnotetext{
${ }^{39}$ Domenico Lo Faso duca di Serradifalco presiede nel 1827 la Commissione di antichità e belle arti con giurisdizione in Sicilia.

${ }^{40}$ Vicari N., 1998: 21.

${ }^{41}$ Vicari N., op. cit.: 24-25.
} 


\section{Bibliografia}

Alajmo, A. G., Matteo Carnilivari e l'architettura del Quattrocento in Agrigento, Riv. Giglio di Rocca, n.18, (1962), pp. 19-25

Bosco, G., Il Comune di Agrigento nel Medio Evo, Agrigento, Edizioni Dioscuri, (1973).

De Gregorio, D., La chiesa agrigentina. Notizie storiche. Vol. I. Dalle origini al secolo XVI, Agrigento, (1996).

Di Fede, M. S., “Agrigento nel Quattrocento”, in M. R. Nobile (a cura di), Matteo Carnilivari Pere Compte 1506-2006, due architetti del gotico nel Mediterraneo, Palermo, Edizioni Caracol, (2006), pp. 6575.

Di Giovanni, G., Agrigento medievale. "Città magnifica". 1087-1492, Palermo, Edizione ZeroNove25, (1997).

De Miro, E., La Valle dei Templi, Palermo, Sellerio Editore, (1994).

Inzerillo, L. "Il gotico chiaramontano e aragonese nella Sicilia occidentale: geometrie a confronto", in M. D'Alessandro (a cura di): L'architettura di età aragonese nell'Italia centro-meridionale. Verso la costruzione di un sistema informativo territoriale documentario e iconografico. L'architettura di età aragonese nel Val di Mazara, Palermo, Edizioni Caracol, (2004), pp. 35-80

Mercurelli, C., "Agrigento Paleocristiana. Memorie storiche e monumentali", in Atti della Pontificia Accademia Romana di Archeologia, Serie III, Memorie, vol. VIII, Agrigento, Tipografia Poliglotta Vaticana, (1948), pp. 26-43.

Miccichè, C., Girgenti. Le pietre della meraviglia...cadute. Osservazioni, note autentiche, documenti editi e inediti per il recupero del Centro storico di Agrigento, Agrigento, (2006).

Peri, I., Per la storia della vita cittadina e del commercio nel Medio Evo. Girgenti porto del sale e del grano, Milano, A. Giuffrè Editore, (1962).

Picone, G., Memorie storiche agrigentine, Ristampa anastatica dell'edizione del 1866 per Stamperia Provinciale Commerciale di Salvatore Montes, Girgenti 1866, a cura del Comune di Agrigento in collaborazione con il Banco di Sicilia, Agrigento, Industria grafica Sarcuto, (1982).

Revenga Domínguez P., "El espectáculo del poder: entradas reales en el Toledo dieciochesco", en Gustavo Curiel (Ed.): Amans Artis. Amans veritatis, México, Instituto de Investigaciones EstéticasUNAM, (2011), pp. 497-544.

Revenga Domínguez, P.: "Metodologías, interpretaciones y tributos de la Historia del Arte". En Palacio Prieto, J.L. (coord.): 90 años de cultura. México: UNAM, (2012), pp. 87-126.

Sardina, P., Il labirinto della memoria. Clan familiari, potere regio e amministrazione cittadina ad Agrigento tra Duecento e Trecento, Caltanissetta-Roma, Salvatore Sciascia Editore, (2011).

Schubring, J, Topografia storica di Agrigento, Ristampa anastatica dell'edizione del 1887 per Ermanno Loescher Editore, Torino, a cura della Casa Editrice Il vespro, Palermo, (1978) (traduzione italiana di G. Toniazzo).

Sutera, D. I Committenti, in Matteo Carnilivari Pere Compte 1506-2006, due architetti del gotico nel Mediterraneo, a cura di M. R. Nobile, Palermo, Edizioni Caracol, (2006).

Vicari, N., L'enigma del tempio di Giove Polieo di Agrigento, Palermo (1998).

Vicari, N., La chiesa di S. Maria dei Greci ad Agrigento, le sue trasformazioni e gli ultimi restauri, Palermo, (2005).

Zalapì, A., "Il maestro degli affreschi di Santa Maria dei Greci ad Agrigento: proposte per una committenza Pujades", in M. C. Di Natale (a cura di), Storia, critica e tutela dell'arte nel Novecento. Un'esperienza siciliana a confronto con il dibattito nazionale, atti del convegno internazionale in onore di Maria Accascina, Caltanissetta, Salvatore Sciascia Editore, (2007), pp. 386-393. 
Zarbo, F., Dal paganesimo al cristianesimo: l'adattamento degli edifici pagani in Sicilia in età medievale, Tesi di dottorato in "Conservazione dei Beni architettonici"- XXIII ciclo, coordinatore del corso Prof. Arch. Stella Casiello, tutor Prof. Arch. Franco Tomaselli, Università degli Studi di Napoli Federico II, Facoltà di Architettura, Dipartimento di storia dell'architettura e restauro, A. A. (2009/2010), Napoli. 